\title{
A wie Alter oder «Experience never gets old»
}

\section{Dominik Heim}

PD Dr. med., Facharzt für Chirurgie, Mitglied FMH

Ich bin auf dem Heimweg von Inawashiro, Japan. Dort war der 35. Kongress der SITEMSH. SITEMSH ist die altehrwürdige Société Internationale de Traumatologie et Médicine des Sports d'Hiver, die 1956 in der Schweiz gegründet wurde. Wintersportunfälle waren damals das Thema. Man traf sich in den Alpenländern. Es war die auslaufende Ära der Hickory-Skis, die Sicherheitsbindung war im Kommen, die Schnallenschuhe kamen auf ... Heute trifft man sich auch ausserhalb der Alpenländer. Und wir haben es - auch nach mehr als 50 Jahren - immer noch nicht geschafft, das Problem der vorderen Kreuzbandruptur im modernen Skisport zu lösen! Auf dem Flug bin ich bei der Filmsuche wahrscheinlich weniger wegen des Themas als wegen Robert de Niro (und Anne Hathaway) auf den Intern mit dem Slogan «Experience never gets old" gestossen. Doch dann zappte ich wegen des Themas nicht weiter! Es geht darin um die Zeit nach der Pensionierung. Jene Zeit also, in der man von der AHV-Preisreduktion profitiert, vom Swiss Pass der SBB über die Tageskarte bei den Bergbahnen bis hin zum Kinoeintritt (ausser montags!).

Einfach ist diese Übergangsphase vom Vollzeitbeschäftigten in die Gesellschaft der AHV-Bezüger nicht. Man mag zwar einen Plan haben, aber der geht nicht immer auf. John Lennon sagte, dass das Leben das ist, was passiert, während du damit beschäftigt bist, Pläne zu machen. Rückblickend war die Nichtwahl zum Generalsekretär einer medizinischen Gesellschaft ein Segen: Die jetzige Arbeit bei der Suva im Team der Versicherungsmedizin erwies sich als äusserst anspruchsvoll und ist eine tägliche intellektuelle Herausforderung. Und eine solche Herausforderung sei ja Prophylaxe der vielleicht einmal anstehenden Altersdemenz. Wie sagte doch Ozzy Osborne von Black Sabbath so treffend: "Of all the things I've lost I miss my mind the most.» Auch der Wechsel in die nahe Privatklinik war ein Glücksfall. Und dass nun eine neu eröffnete Permanence im Nobelort der Berner Alpen an meinen Fachkenntnisssen interessiert ist, das schmeichelt. Man wird in dieser AHV-Phase nicht mehr nach dem master of ... qualifiziert, sondern nach der persönlichen «Experience», und eine solche kann nach vielen klinischen Jahren doch ganz beachtlich sein. Dass man jetzt eher die C6-Varizen als die schönen C2-Beine zur Behandlung kriegt, verwundert also nicht. Die ACLuxation beim Snowboarder sieht man schon durch das T-Shirt hindurch noch vor dem Röntgenbefund, und bei der Segondfraktur weiss man schon zum Vornherein, was das MRI dann zeigen wird. Experience! Auch De Niro besitzt sie im Intern: Als 70-jähriger Witwer, charmant, mit Krawatte und Anzug, zurückhaltend und erfahren, steigt er als Praktikant in ein E-Commerce-Modeunternehmen ein. Pragmatisch sieht er im Alltag die Lösung und nicht das Problem. The Intern ist ein Vergnügen, typisch Hollywood-geschönt. Schön, dass die Ehe der arbeitenden Anne Hathaway mit ihrem Mann als Hausmann doch noch gerettet werden kann, sympathisch, dass die Zerreissprobe zwischen beruflicher Karriere, Family Commitment, und Fremdgehen in "Pretty-Woman»-Manier doch noch ein Happy End findet.

Mediziner werden auch pensioniert. Mit 65 scheidet man üblicherweise in öffentlichen Betrieben aus, und viel Erfahrung liegt brach. Deshalb denkt man z.B. bei der Suva über die Möglichkeit einer Weiterbeschäftigung nach dem offiziellen Pensionierungsalter nach. Erfahrung weitergeben, aber einen beruflichen Tapetenwechsel machen und nochmals etwas Neues in Angriff nehmen, weniger angebunden sein als früher, aber nochmals die Herausforderung suchen. Und es wird geschätzt: «Das klingt jetzt vielleicht blöd, aber ich mag ältere Menschen», sagt Anne Hathaway im Interview.

Eine Idee hat sich auf dem Flug (und schon etwas vorher) in meinem Kopf festgesetzt: Es wäre wichtig, die SITEMSH nach all den Jahren wieder für einen Wintersportkongress in die Schweiz zu «holen». Da hat es viel «Experience» zum Weitergeben, und schliesslich ist auch am Kongress in Japan die Lösung fürs Kreuzband immer noch nicht gefunden worden ... 